\title{
Using the 3D Body Scanner in Elite Sports
}

\author{
Axel SCHÜLER ${ }^{1}$, Ina FICHTNER, Olaf UEBERSCHÄR \\ Institute for Applied Training Science, Leipzig University, Leipzig, Germany
}

DOI: $10.15221 / 18.216 \quad$ http://dx.doi.org/10.15221/18.216

\begin{abstract}
Using laser-based 3D body scanners in elite sports may offer a decisive advantage with respect to individual motion optimization and training. In the following, a brief overview of various applications of 3D body scanning in elite sports will be given as employed at the Institute for Applied Training Science. In many artistic sports, such as figure skating, gymnastics or diving, high rotation speeds for twists and somersaults are required for successful competition performance. To achieve those high angular velocities in air, athletes must adopt minimal moments of inertia (MOI) with respect to the rotational axis. 3D body scanners can easily be used to measure $\mathrm{MOI}$ and detect even small changes between different individual postures. Thus, optimal individual rotation positions for twists and somersaults can be determined. Five straight positions and four tucked positions were compared with respect to their $\mathrm{MOI}$ around the longitudinal and mediolateral axes, respectively. Compared to the standard up-right standing position, we were able to show that a straight position with forearms crossed in front of the chest yields a $12 \%$ smaller $\mathrm{MOI}$ for the longitudinal axis. Regarding the mediolateral axis, a face-down tucked position generates an up to $30 \%$ smaller $\mathrm{MOI}$ than a face-up tucked position. Moreover, for figure skating not only an optimal arm position but also closing the knees and twisting the shoulder and hip portions contribute to a significant decrease in MOI.

In ski jumping and snowboard cross, on the other hand, minimal aerial drag is a key performance factor. Employing 3D body scanner measurements there, aerodynamically unsuitable clothing can be identified. For ski jumping, 3D scans can also be used to reveal disadvantageous in-run postures, e.g. too big knee angles or aerodynamically suboptimal head, back or hand positions. Finally, anthropometric data of athletes as derived from body scanner measurements are also used for purposes of motion analysis and biomechanical simulations.
\end{abstract}

Keywords: motion analysis, acrobatic sports, moments of inertia, aerial drag, anthropometry

\section{Introduction}

In this review we give a brief summary of our research in field of body scanning for elite sports for the past five years. This paper is divided into two parts: In the first part, we compare commonly used geometric human body models to scanner-derived human body models with respect to segment mass distribution and the total inertia tensor. In the second part it is demonstrated how we apply bodyscanner-derived individual body models to improve technical performance in acrobatic and artistic sports, such as gymnastics and figure skating, as well applications in ski jumping.

The knowledge of the mass-geometric properties of body segments is indispensable for valid motion analysis and simulations of human motion in the research fields of sports biomechanics, orthopedics and ergonomics. A comprehensive overview of the history of studying human body segment parameters (BSP) is given in [1]. In the past, the most popular human body models were those derived by Dempster, Clauser, Hanavan and Zatsiorsky [2-5]. Nowadays, the body model of de Leva [6] is best established, representing basically an enhanced modification of Zatsiorsky's model.

A BSP model is characterized by three groups of parameters [7], namely the mass of the segments, the position of their barycenters and the moments of inertia (MOI), including the principal $\mathrm{MOI}$ and their axes.

Along with the rise of laser technology in everyday-life in the 1990's, a new, precise, user-friendly device for computer-assisted anthropometric measurements was developed: The so-called body scanner (or laser scanner) produces a 3D image of the athlete's surface based on the principle of laser triangulation. Using Poisson surface reconstruction [8], a closed triangular mesh is created, enabling the computation of volumes, surface areas, volume center coordinates and inertia tensors of the whole body as well as of individual segments. By convention, we refer to a body scan model if explicitly the whole surface mesh is known and used. Otherwise, i.e. if only single segment or arc lengths are used, we speak of geometric human models. 
In all studies, we used the whole body laser scanner VITUS Smart XXL (Human Solutions GmbH, Kaiserslautern, Germany) and the software AnthroScan 3.0.1. In [9] we compared anthropometrically individualized Hanavan models [4] to a body scan model with respect to mass distribution and moments of inertia, the latter being accessible to the sports scientist in terms of observed angular momenta. In [10] we computed and compared principal MOls on the basis of three different models: Two geometric models are based on Zatsiorsky, one universal model with only three parameters and one individual model with 35 length parameters and one body scan model.

In $[11,12,13]$ we focused on applications in gymnastics and figure skating. Since strength capabilities of the athlete are generally limited, so are flight height and generation of angular momentum. Effective flights in those sports are characterized by very small mean moments of inertia. One option to achieve those is to reduce the moment of inertia as quickly as possible after take-off, aiming for a tightly closed rotational position. Similarly, the technique advancement from low to high revolution jumps in figure skating is facilitated by both the increase of rotational velocity and the decrease of $\mathrm{MOI}$ [14].

\section{Methods}

\subsection{Individualization of standard human body models}

\subsubsection{Customizing an alaska/Dynamicus model by means of $3 D$ body scans}

Anthropometric data derived from body scanner measurements are used to customize simulationenvironment body models, in our case an alaska/Dynamicus model (Institute of Mechatronics, Chemnitz, Germany, 2016), see Figure 1 (Left, Center). Thirty-five length and circumference values are used to define the human "Dynamicus" model in the alaska modelling environment. In particular, a simplified body skeleton, the so-called skeleton function of AnthroScan (Human Solutions GmbH, Kaiserslautern, Germany), is used to determine the lengths of the upper and lower limbs. A semi-automatic segmentation function also provides the volumes of relevant body segments and, by assuming constant density, also their masses.
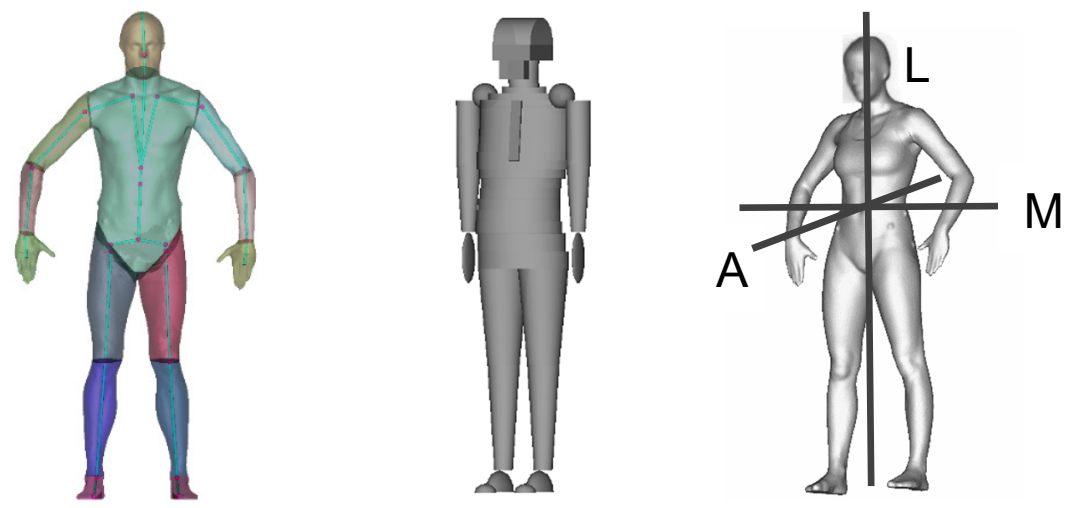

Figure 1. Left: $3 D$ surface mesh with segmentation and the simplified skeleton (VITUS Smart XXL, Human Solution). Center: alaska/Dynamicus model consisting of 44 segments (Institute of Mechatronics, Chemnitz). Right: Longitudinal, mediolateral, and anteroposterior axes (L, $M, A)$.

Furthermore, the moments of inertia of the total body are computed. The principal axes for a human body in an erect standing position are the longitudinal axis, the mediolateral axis (i.e. from left to right) and the anteroposterior axis (i.e. from front to rear), as shown in Figure 1 (Right).

\subsubsection{Comparison of individualized models: Mass distribution and angular momenta}

In [9] we computed angular momenta during take-off in diving in two different ways: First, we used the Hanavan model; second, the body scan model was employed. In total, three divers were recorded, each in 13 different variations of take-off motion for a backward somersault. We could show that both models yielded almost identical values of the total angular momentum. However, the contribution of single segments turned out to differ by up to $5 \%$, especially in the case of the head or the arms.

\subsubsection{Comparison of individualized models: Moments of inertia}

In [10] we compared three different human models with respect to their principal moments of inertia. In this study, 17 athletes (8 male, 9 female) participated and were scanned in several upright erect positions (altogether 34 scans) and, additionally, in compact, tucked, "piked" and sitting positions (25 scans). It was shown that for erect positions the mean difference of the moments of inertia of the individual models and the 3D scanner model is zero. The corresponding standard deviation with respect to the mediolateral and anteroposterior axes is about $2 \%$, while it amounts to approximately $8 \%$ as for the longitudinal axis. 


\subsection{Applications to gymnastics}

In a further study [11] two male athletes participated $(A 1: 60.5 \mathrm{~kg}, 1.61 \mathrm{~m}, 33 \mathrm{yrs}$ and $\mathrm{A} 2: 70.0 \mathrm{~kg}$, $1.77 \mathrm{~m}, 36 \mathrm{yrs}$ ). Each was scanned in five standing and four tucked positions, see Figure 2 . The inertia tensor of the mesh volume was computed using MeshLab (version 1.3.3, Visual Computig Lab, SourceForge). Moreover, the principal moments of inertia were computed (with respect to the mass center) as well as the rotation matrix $S$, which transforms the principal inertia matrix $J=\operatorname{diag}\left(J_{1}, J_{2}, J_{3}\right)$ in terms of body coordinates into the inertia matrix $S=S J S^{\top}$ in terms of global laboratory coordinates. We distinguished two groups of tuck positions: one group with open knees and one with closed knees. In each group, we studied a face-down and a face-up head position.

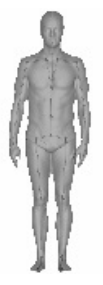

S1

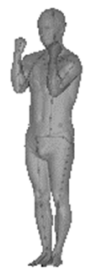

S2

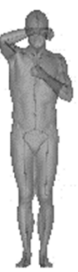

S3

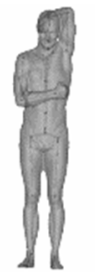

S4

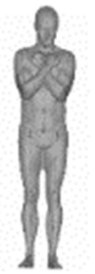

S5

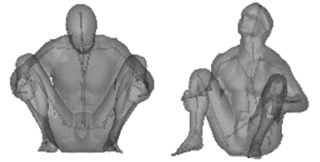

$\mathrm{T} 1$

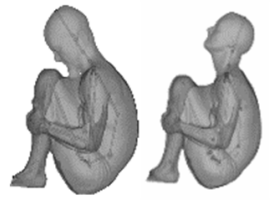

T4

Figure 2. Left: five standing positions S1,.., S5. Right: four tucked positions T1,.., T4.

Special care is required for the tucked positions: Computing the closed mesh in AnthroScan, undesired mesh-merging appears in regions with low point density and self-intersection of the surface, see Figure 3 . The merged mesh volume $V_{1}$ is thereby up to $10 \%$ bigger than the (exact) standard volume $V_{0}$ of pose $\mathrm{S} 1$. To correct for this error to first approximation, we apply a proportional scaling of the inertia matrix, yielding $J_{\text {corr }}=J V_{0} / V_{1}$.

A second correction step considers the mass. Since MeshLab by default uses the standard density $\rho_{\mathrm{ML}}=1 \mathrm{~kg} / \mathrm{m}^{3}$, we apply the scaling factor $\rho_{\mathrm{ML}} / \rho_{0}$ where $\rho_{0}=\mathrm{m} / V_{0}$ denotes the mean density of the gymnast, with $m$ denoting the mass and $V_{0}$ the volume in position $S 1$.
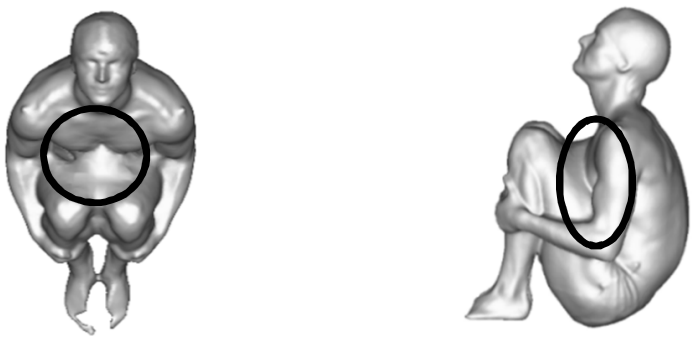

Figure 3. Undesired mesh-merging between thighs and breast (oval marking) in tucked positions.

\subsection{Applications to figure skating}

In the studies [12, 13], five male elite figure skaters $A 1, \ldots, A 5$ (mass $=73.5 \pm 10.6 \mathrm{~kg}$, height $=178 \pm 8 \mathrm{~cm}$ and age $=23.4 \pm 2.1 \mathrm{yrs}$ ) took part. They were scanned in seven different flight positions - two closing positions (right after take-off), three closed flight positions (closed feet), and two landing positions (just after first re-contact on ice), see Figure 4. Moments of inertia were compared and the best position in each group was identified. Based on those results, a quantitative rotation benefit was determined in terms of "additionally achieved" degrees per rotation.

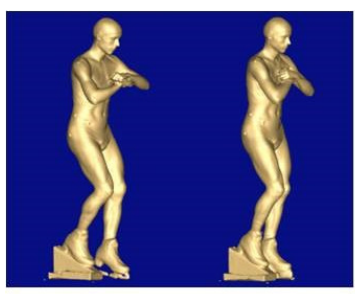

(a) closing

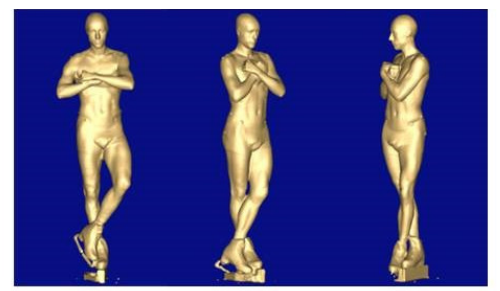

(b) closed flight

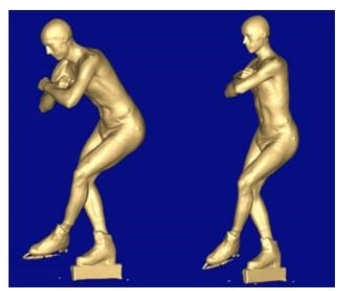

(c) landing

Figure 4. Three different phases of flight in figure skating: closing, closed flight, and landing. 


\section{Results}

\subsection{Applications to gymnastics}

\subsubsection{Upright positions}

Since athlete $A 2$ is taller and heavier than A1, the absolute values of his longitudinal moments of inertia are bigger than those of $A 1$. However, for both athletes $A 1$ and $A 2$ standing positions S1, S2, and S3 possess almost the same moments of inertia (A1: $\left.0.75 \mathrm{~kg} \mathrm{~m}^{2}, \mathrm{~A} 2: 0.98 \mathrm{~kg} \mathrm{~m}^{2}\right)$, see Figure 5 .

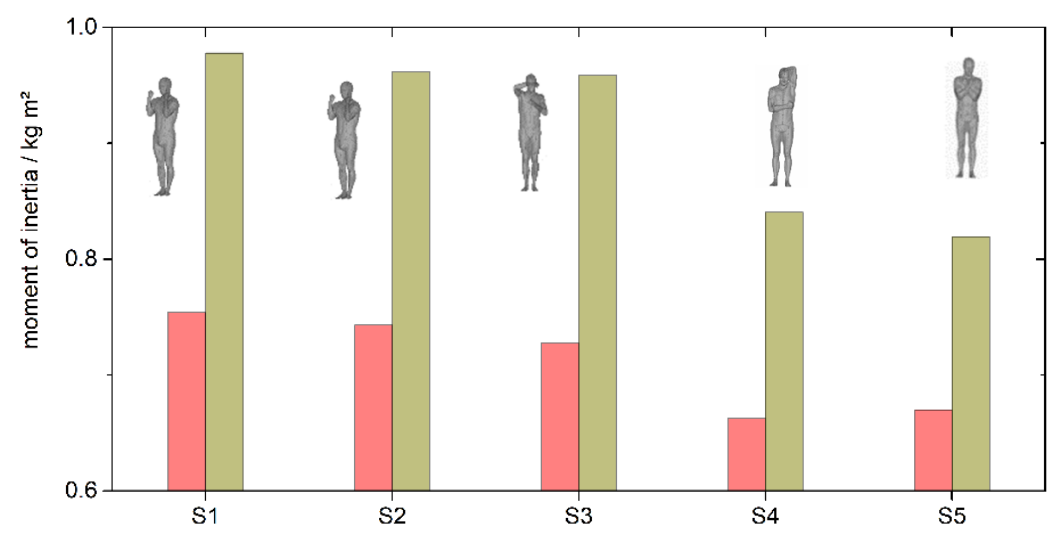

Figure 5. Moments of inertia w.r.t. the longitudinal axis for standing Positions S1 to S5. red: A1, green: A2.

Postures S4 and S5 have $9 \%$ (A1) and $12 \%$ (A2) smaller moment of inertia than S1. Consequently, for twists, gymnasts should prefer rotation positions S4 and S5 rather than S1, S2 or S3.

\subsubsection{Tucked positions}

For both athletes $\mathrm{A} 1$ and $\mathrm{A} 2$ the tucked positions $\mathrm{T} 1$ and $\mathrm{T} 3$ (face-down) obviously yield a smaller moment of inertia than T2 and T4 (face-up), see Figure 6. However, there is almost no difference between open and closed knees. Both postures T1 and T3 show the same moments of inertia.
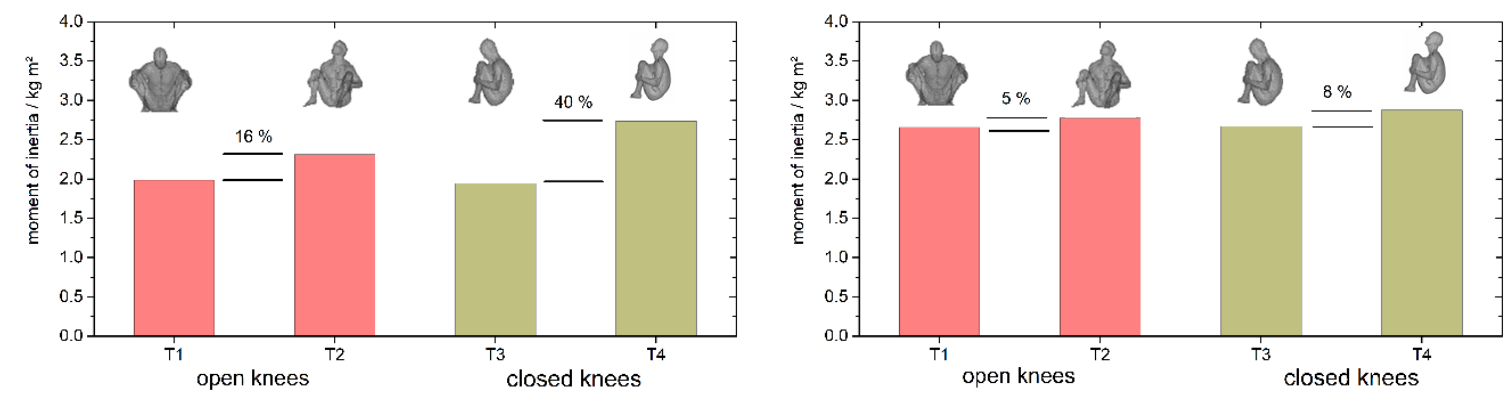

Figure 6. Comparison of moments of inertia for tucked positions T1,.., T4. Left: A1. Right: A2.

Since A2 has a greater mass than A1, moments of inertia are bigger. The smaller inertia differences $(5 \%$ and $8 \%)$ for A2 may come from less accentuated posing.

\subsection{Applications to figure skating}

It was shown that a closed flight position with closed knees (FP2) or with twisted hip and shoulders (FP3) yield up to $17 \%$ smaller moments of inertia than flight position with open knees and elbows outside (FP1), see Figure 7. 


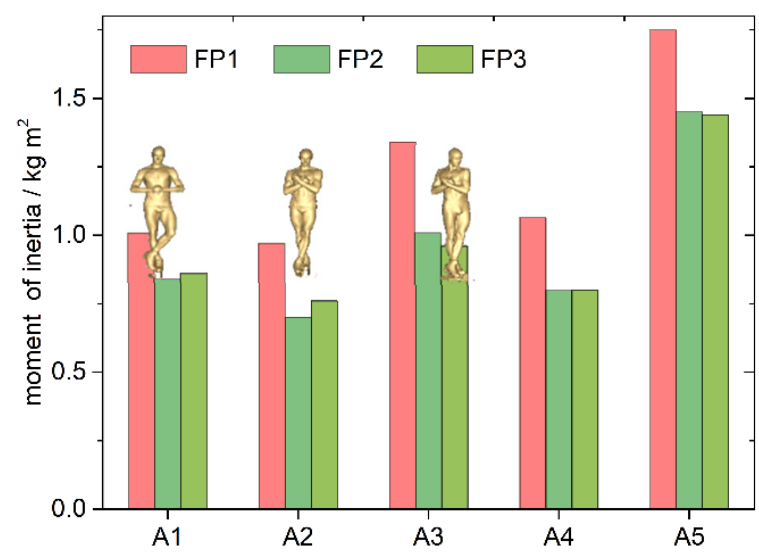

Figure 7. Moments of inertia in a closed flight position. Comparison of positions FP1, FP2, and FP3 for athletes A1,.., A5.

As for practical sports application, we identified the closed flight positions FP2 and FP3 (green), with arms tightly to the body and elbows in front, as the preferable positions since they have the smallest moments of inertia. Flight position FP1 (red) with closed feet but open knees and elbows outside, is not recommended for quadruple jumps because of higher moment of inertia. For all five athletes FP1 implies a distinctly higher moment of inertia than FP2 and FP3.

\subsection{Applications to ski jumping}

A ski jumper tries to maintain an optimal aerodynamic position during the in-run. To reduce air resistance as well as canting, German female athletes were scanned in their individually preferred positions.
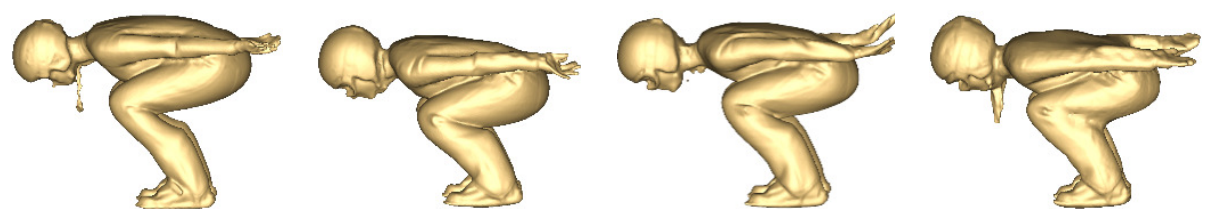

Figure 8. In-run positions of athletes $A 1, \ldots, A 4$, side view.

The ski jumpers showed different knee angles (Figure 8), resulting in different trunk positions. A1 exhibits a disadvantageous back position, resulting in higher drag. Moreover, the hand positions of $A 1$ and A4 are adequate whereas A3's hand position is suboptimal.
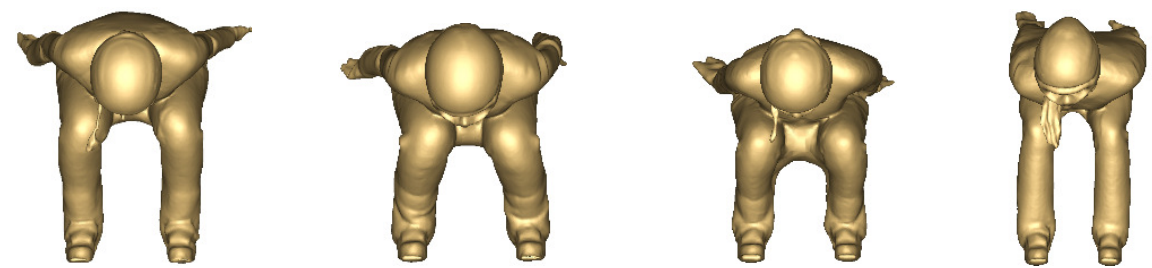

Figure 9. In-run positions of athletes $A 1, \ldots, A 4$, front view.

Finally, also a parallel shank position is essential to avoid additional frictional forces during in-run (Figure 9).

\section{Discussion and Conclusions}

In $[9,10]$ it was shown that the studied geometric human models (universal or individual) yield almost the same results as a body scanner mesh model with respect to computing motion parameters. That equivalence of the models was shown both indirectly via biomechanical analysis of 39 take-off motions in diving as well as directly via comparing principal moments of inertia in compact and straight positions. The assumption of homogeneous mass distribution produced only little error [15]. The body scan model has proven useful to get a reliable individual human body model. 
In $[12,13]$ the body scan mesh surface turned out to be a helpful tool to compute and compare principal moments of inertia. Especially in elite sports, it is essential for performance enhancement to detect minimal differences: What are the optimal arm, leg shoulder-hip and knee positions for a successful quadruple jump in figure skating? What are the best hand, feet, and head positions for a triple somersault tuck? Body scanner measurement discloses the disadvantages of wrong rotational positions. The benefit of an optimal position can be quantified as an increase of rotational speed.

\section{Acknowledgements}

This research is part of a project funded by the German Federal Ministry of the Interior, Building and Community on the basis of a resolution of the German Bundestag.

We thank our colleagues Dr. Karin Knoll, Dr. Klaus Knoll, Angelika Kretschmer, Dr. Thomas Lehmann, and Dr. Sören Müller for their support in the measurements, the processing of biomechanical parameters and useful discussions.

\section{References}

[1] Pearsall, D. J., \& Reid, G. (1994). The study of human body segment parameters in biomechanics. Sports Medicine, 18(2), 126-140.

[2] Dempster, W. T. (1955). Space requirements of the seated operator. Wright-Patterson Air Force Base, Ohio.

[3] Clauser, E. P., McConville, J.T. \& Young, J.W. (1969). Weight, volume and center of mass of segments of the human body. Aerospace Medical Research Laboratories technical report, WrightPatterson Air Force Base, Ohio.

[4] Hanavan, E. P. (1964). A mathematical model of the human body. Ohio: Aerospace Medical Research Laboratories, Wright-Patterson Air Force Base, Ohio.

[5] Zatsiorsky, V. M. and Seluyanov, V. N. (1985). Estimation of the mass and inertia characteristics of the human body by means of the best predictive regression equations. Biomechanics IX-B (Edited by Winter, D. A., Norman, R. W., Wells, R. P., Hayes, K. C. and Patla, A. E.), pp. 233-239. Champaign, IL: Human Kinetics.

[6] de Leva, P. (1996). Adjustments to Zatsiorsky-Seluyanov's segment inertia parameters. J. Biomechanics, 29 (9), 1223-1230.

[7] Hatze, H. (1980). A mathematical model for the computational determination of parameter values of anthropometric segments. J. Biomechanics 13, 833-843.

[8] Bolitho, M., Kazhdan, M., Burns, R. \& Hoppe, H. (2009). Parallel poisson surface reconstruction. In G. Bebis et al. (Eds.), Advances in Visual Computing, Lecture Notes in Computer Science, vol 5875, 678-689. Berlin, Heidelberg: Springer.

[9] Schüler, A., Kerner, S. \& Witt, M. (2015). A Comparison of two body segment parameter models via angular momentum at takeoff in diving, In: Colloud, F., Domalain, M., and Monnet, T: (eds.), eProceedings of the 33rd Conference of the International Society of Biomechanics in Sports, 2015, pp. 146-149, ISSN 1999-4168, Poitiers: France, Poitiers University.

[10] Schüler, A., Dee, R., Knoll, K., Ueberschär, O. \& Fichtner, I. (2016). Berechnung von Trägheitsmomenten anhand von Bodyscans - ein Methodenvergleich. In K. Witte, N. Bandow \& J. Edelmann-Nusser (Hrsg.), Sportinformatik XI, 88-95. Aachen: Shaker.

[11] Knoll, K., Schüler, A. \& Lehmann, T. (2016). Effective positions for rotations about the longitudinal axis - an example in figure skating jumps. In M. Ae, Y. Enomoto, N. Fujii \& H. Takagi (Eds.), Eproceedings of the 34th ISBS Symposium 2016, 187-190. Tsukuba: University of Tsukuba

[12] Schüler, A., Dee, R., Ueberschär, O. \& Fichtner, I. (2017). Vergleich von Massenträgheitsmomenten gestreckter und gehockter Posen in akrobatischen Sportarten. In M. Rauscher \& M. Witt (Eds.), dvs-Tagung Biomechanik, Biomechanik und Kognition (Leipziger Sportwissenschaftliche Beiträge, 14, pp. 69-75). Berlin: Lehmanns Media.

[13] Schüler, A., Dee, R., Ueberschär, O. \& Fichtner, I. (2017). Minimal moments of inertia - an analysis of optimal twist and somersault positions, Proceedings of ECSS MetropolisRuhr 2017.

[14]King, D., Smith, S., Higginson, B., Muncasy, B., \& Scheirman, G. (2004). Figure Skating: Characteristics of triple and quadruple toe-loops performed during the Salt Lake City 2002 Winter Olympics. Sports Biomechanics, 3 (1), 109-123.

[15] Ackland, T. R., Henson, P. W., \& Bailey, D. A. (1988). The uniform density assumption: its effect upon the estimation of body segment inertial parameters. International Journal of Sport Biomechanics, 4 (2), 146-155. 